\title{
A POTENTIAL ENERGY OF INTERACTION BETWEEN HELIUM ATOMS
}

\author{
I. O. Vakarchuk, V. V. Babin, A. A. Rovenchak \\ Ivan Franko National University of Lviv, Department of Theoretical Physics \\ 12 Drahomanov Str., UA-79005, Lviv, Ukraine
}

(Received November 5, 1999)

\begin{abstract}
In the work the pair potential of interatomic interaction between helium atoms is established from the first principles without using fitting parameters. Previously obtained from Schrödinger equation connection between the potential Fourier image and the coefficient functions in the expansion of the many-boson system ground state wave function logarithm by the "degrees" of density fluctuations are used. RPA as the zero approximation and the approximation of "two sums over wave vector" as the first one are considered.

The results of this work lead to the value of the first sound velocity of $231 \mathrm{~m} / \mathrm{s}$ while the experiment gives $237 \mathrm{~m} / \mathrm{s}$. The fit of our potential with Aziz model potential is quite good: the well depths are $-10.58 \mathrm{~K}$ and $-11.04 \mathrm{~K}$ respectively, the minimum positions being $3.34 \AA$ and $2.99 \AA$.

Key words: liquid helium, interatomic potential, random-phase approximation, first sound.
\end{abstract}

PACS number(s): 05.30.Jp, 34.20.Cf, 67.40.Mj, 71.45.-d

\section{INTRODUCTION}

When developing a theory of many particle systems from the first principles a key value in terms of the initial information is seen in the potential energy which predetermines the nature of the inter-particle interaction. It is generally assumed that it has a pair nature and is expressed through the sum of all the pair interactions between the particles. When speaking about the pair potential we virtually mean a certain effective twoparticle interaction leading to an agreement between the theoretical results and the observed, yet fully neglected, many-particle interactions whose contribution into the thermodynamic functions of the system is known to be quite substantial in some cases $[1,2]$.

There are several ways of establishing the potential energy. Firstly, we can accomplish this task via a direct quantum mechanics calculation. Secondly, we can accept a specified model form of the potential whose parameters are to be determined by an agreement between the calculated and observable values of physical quantities [3-5]. Another possibility arises when solving the inverse problem within the theory of particles scattering. Should the latter be coming into effect on the condensed matter bodies, the potential is recoverable by the experimental values of the so-called structure factor.

Within the classical approach $[1,2]$ proceeding from the pair structure factor $S_{q}$ of the $N$ particles system it is easy to obtain a radial distribution function

$$
F_{2}(r)=1+\frac{1}{N} \sum_{\mathbf{q} \neq 0}\left(S_{q}-1\right) \exp (-i \mathbf{q r})
$$

We can further obtain the $\Phi(r)$ pair inter-particle po- tential in certain approximations by the known function $F_{2}(r)$ from Bogoliubov-Born-Green-KirkwoodIvon equation. Studies of this type were carried out for simple liquids such as argon or for liquid metals [2]. In the case of simple liquids a potential of the Lennard-Jones type was obtained, the so-called long-range interaction oscillating potentials having been received for liquid metals.

Reproducing the $\Phi(r)$ inter-particle interaction pair potential in ${ }^{4}$ He from the experimental values of the $S_{q}$ pair structure factor seems quite an interesting problem to solve. The classical scheme is understandably unfit for low temperatures owing to the fact that in this region ${ }^{4} \mathrm{He}$ is to be interpreted in terms of quantum mechanics.

\section{INITIATING EXPRESSIONS}

Our task consists in rediscovering $\Phi(r)$ from $S_{q}$ calculated experimentally from the scattering of heat neutrons in the superfluid ${ }^{4} \mathrm{He}[8]$ within the framework of the quantum mechanics description. For this purpose one must possess a connection between the Fourier image of the pair interaction potential $\nu_{q}$ and $S_{q}$ which is far more complex than in the classic case. The solution of this problem in the two sums approximation over the wave vector follows from the results obtained in $[6,7]$ where a many-boson non-ideal system is considered at $T=0 \mathrm{~K}$ (i.e. in the ground state from the point of view of quantum mechanics). In [7] a sequence of equations was obtained for the coefficient functions $a_{2}, a_{3}, a_{4}, \ldots$ in the expansion of the many-boson system ground state wave function logarithm by the "degrees" of collective variables:

$$
\Psi\left(\mathbf{r}_{1}, \ldots, \mathbf{r}_{N}\right)=C \exp \left\{\sum_{n \geq 2} \frac{N^{1-n / 2}}{n !} \sum_{\mathbf{k}_{1} \neq 0} \ldots \sum_{\mathbf{k}_{n} \neq 0} \delta\left(\mathbf{k}_{1}+\ldots+\mathbf{k}_{n}\right) a_{n}\left(\mathbf{k}_{1}, \ldots, \mathbf{k}_{n}\right) \rho_{\mathbf{k}_{1}} \ldots \rho_{\mathbf{k}_{n}}\right\}
$$




$$
\rho_{\mathbf{k}}=\frac{1}{\sqrt{N}} \sum_{j=1}^{N} e^{-i \mathbf{k} \mathbf{r}_{j}}
$$

In the approximation of "two sums over the wave vector" we shall receive the following system of equations from Schrödinger equation (1-3):

$$
\begin{aligned}
& \begin{array}{l}
\frac{N}{V} \frac{m \nu_{k}}{\hbar^{2}}+\mathbf{k}^{2} a_{2}(\mathbf{k})-\mathbf{k}^{2} a_{2}^{2}(\mathbf{k})-\frac{1}{2 N} \sum_{\mathbf{q} \neq \mathbf{0}} \mathbf{q}^{2} a_{4}(\mathbf{q},-\mathbf{q}, \mathbf{k},-\mathbf{k})+\frac{1}{N} \sum_{\mathbf{q} \neq \mathbf{0}} \mathbf{q}(-\mathbf{k}-\mathbf{q}) a_{3}(\mathbf{k}, \mathbf{q},-\mathbf{k}-\mathbf{q})=0, \\
a_{3}\left(\mathbf{k}_{1}, \mathbf{k}_{2}, \mathbf{k}_{3}\right)=\frac{-2}{\mathbf{k}_{1}^{2}\left[1-2 a_{2}\left(\mathbf{k}_{1}\right)\right]+\mathbf{k}_{2}^{2}\left[1-2 a_{2}\left(\mathbf{k}_{2}\right)\right]+\mathbf{k}_{3}^{2}\left[1-2 a_{2}\left(\mathbf{k}_{3}\right)\right]} \\
\quad \times\left\{\mathbf{k}_{1} \mathbf{k}_{2} a_{2}\left(\mathbf{k}_{1}\right) a_{2}\left(\mathbf{k}_{2}\right)+\mathbf{k}_{1} \mathbf{k}_{3} a_{2}\left(\mathbf{k}_{1}\right) a_{2}\left(\mathbf{k}_{3}\right)+\mathbf{k}_{2} \mathbf{k}_{3} a_{2}\left(\mathbf{k}_{2}\right) a_{2}\left(\mathbf{k}_{3}\right)\right\}, \\
a_{4}(\mathbf{k},-\mathbf{k}, \mathbf{q},-\mathbf{q})=\frac{1}{\mathbf{k}^{2}\left[1-2 a_{2}(\mathbf{k})\right]+\mathbf{q}^{2}\left[1-2 a_{2}(\mathbf{q})\right]}\left\{(\mathbf{k}+\mathbf{q})^{2} a_{3}^{2}(\mathbf{k}+\mathbf{q},-\mathbf{k},-\mathbf{q})+(\mathbf{k}-\mathbf{q})^{2} a_{3}^{2}(\mathbf{k}-\mathbf{q},-\mathbf{k}, \mathbf{q})\right. \\
\left.+2\left[\mathbf{k}(\mathbf{k}+\mathbf{q}) a_{2}(\mathbf{k})+\mathbf{q}(\mathbf{q}+\mathbf{k}) a_{2}(\mathbf{q})\right] a_{3}(\mathbf{k}+\mathbf{q},-\mathbf{k},-\mathbf{q})+2\left[\mathbf{k}(\mathbf{k}-\mathbf{q}) a_{2}(\mathbf{k})+\mathbf{q}(\mathbf{q}-\mathbf{k}) a_{2}(\mathbf{q})\right] a_{3}(\mathbf{k}-\mathbf{q},-\mathbf{k},+\mathbf{q})\right\}
\end{array}
\end{aligned}
$$

Here we shall skip over the expressions obtained for the $s$-particle density matrices $F_{s}\left(\mathbf{r}_{1}, \ldots, \mathbf{r}_{s}\right)$ through the functions $a_{2}, a_{3}, a_{4}$ from [7]. Hence, taking into account the simple connection of the pair distribution function and the pair structure factor of the system this implies a correlation in the approximation of "the two sums over the wave vector" between $a_{2}, a_{3}, a_{4}$ and $S_{q}$ at the absolute zero temperature:

$$
a_{2}(\mathbf{q})=\frac{1}{2}\left(1-\frac{1}{S_{q}}\right)-\frac{1}{2} \Sigma(\mathbf{q})
$$

where $\Sigma(\mathbf{q})$ is a complex functional of $a_{2}$ :

$$
\begin{aligned}
\Sigma(\mathbf{q})= & \frac{2}{N} \sum_{\substack{\mathbf{k}_{1} \neq 0 \\
\mathbf{k}_{1}+\mathbf{k}_{2}+\mathbf{q}=0}} \sum_{\mathbf{k}_{2} \neq 0} \frac{1}{\left[1-2 a_{2}\left(\mathbf{k}_{1}\right)\right]\left[1-2 a_{2}\left(\mathbf{k}_{2}\right)\right]}\left\{a_{2}\left(\mathbf{k}_{1}\right) a_{2}\left(\mathbf{k}_{2}\right)+a_{3}\left(\mathbf{k}_{1}, \mathbf{k}_{2}, \mathbf{q}\right)\left[1+a_{3}\left(\mathbf{k}_{1}, \mathbf{k}_{2}, \mathbf{q}\right)\right]\right\} \\
& +\frac{1}{N} \sum_{\mathbf{k} \neq 0} \frac{a_{4}(\mathbf{k},-\mathbf{k}, \mathbf{q},-\mathbf{q})}{\left[1-2 a_{2}(\mathbf{k})\right]} .
\end{aligned}
$$

In correlation (4) it will suffice to calculate the value of $\Sigma(\mathbf{q})$ on the functions $a_{2}(\mathbf{q})$ in the random phase approximation

$$
a_{2}(\mathbf{q})=\frac{1}{2}\left(1-\frac{1}{S_{q}}\right)
$$

Next, we shall determine the Fourier image $\nu_{q}$ of the potential in the accepted approximation from (1). For $a_{3}, a_{4}$ it is enough to be confined by the RPA which means that we shall embed the $a_{2}$ value determined from (6) into the right-hand side of (2) and (3). We shall execute the transition into $\Phi(\mathbf{r})$ in a usual way

$$
\Phi(r)=\frac{1}{V} \sum_{\mathbf{q}} e^{i \boldsymbol{q} \mathbf{r}} \nu_{q} .
$$




\section{RESULTS AND THEIR DISCUSSION}

For carrying out numerical calculations we made use of the experimental values of the superfluid ${ }^{4} \mathrm{He}$ structure factor from [8] which correspond to the density value of $\rho=0.02185 \AA^{-3}$ and to the temperature $T=1 \mathrm{~K}$. As the initial expressions contain $S_{q}$ at $T=0 \mathrm{~K}$ we converted these values into those at $T=0 \mathrm{~K}$ falling back on correlation [9]

$$
\begin{aligned}
& S_{q}=S_{q}(T) \tanh \frac{E_{q}}{2 T}, \\
& E_{q}=\frac{\hbar^{2} q^{2}}{2 m S_{q}}, \\
& S_{q}=\left.S_{q}\right|_{T=0},
\end{aligned}
$$

which were treated as equations for $S_{q}$ at the given $S_{q}(T)$. A plot of the smooth structure factor from [8] as well as $S_{q}$ is given in Fig. 1 that corresponds to Table 1.

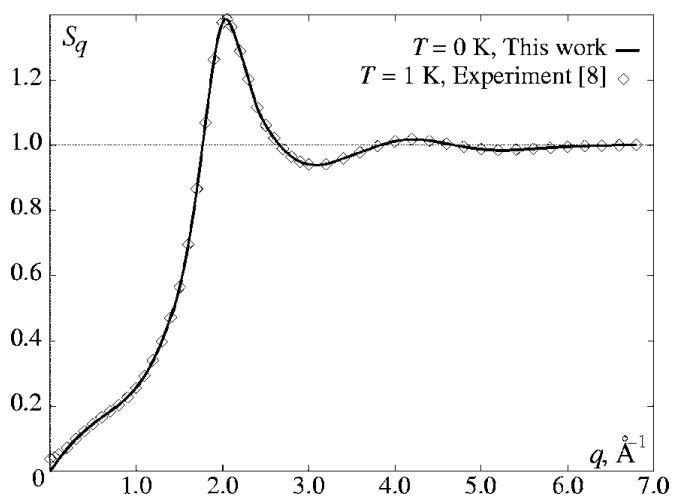

Fig. 1. The ${ }^{4}$ He structure factor.

\begin{tabular}{|c|c|c|c|}
\hline \hline & $r_{\min }, \AA$ & $\Phi\left(r_{\min }\right), \mathrm{K}$ & $\Phi(0), \mathrm{K}$ \\
\hline \hline RPA & 3.31 & -17.08 & $\simeq 242$ \\
\hline 1st approximation & 3.34 & -10.58 & $\simeq 230$ \\
\hline Aziz model potential [5] & 2.99 & -11.04 & $\simeq 2 \cdot 10^{6}$ \\
\hline Lennard-Jones potential [10] & 2.556 & -10.22 & $\infty$ \\
\hline \hline
\end{tabular}

Table 1. Comparison of the results.

Making use of $S_{q}$ received in this fashion we carried out a calculus of $\nu_{q}$ and $\Phi(r)$ in the random phase approximation and the subsequent approximation of "two sums over the wave vector". The random phase approximation implies that it is necessary to discard all the terms which contain the sums over the wave vector from (1) and to take $a_{2}$ from (6), i. e.

$$
\nu_{q}=\frac{\hbar^{2} q^{2}}{2 m} \frac{1}{2 \rho}\left(\frac{1}{S_{q}^{2}}-1\right)
$$

The results of the calculations are adduced in Tables $2-4$ and Figs. 2-4.

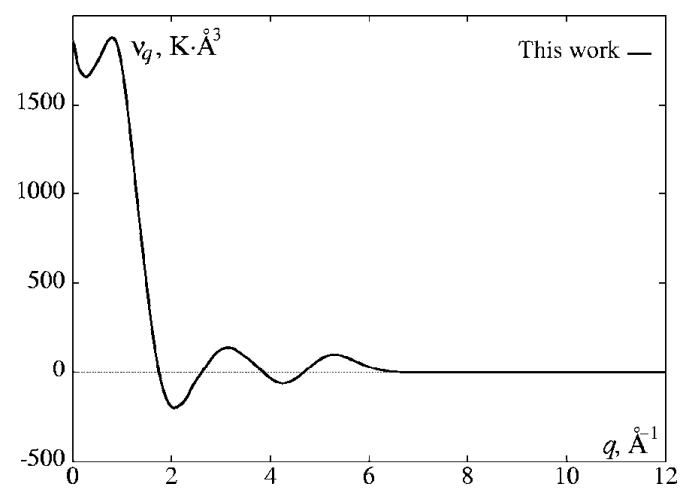

Fig. 2. The Fourier image of the potential of interatomic interaction in ${ }^{4} \mathrm{He}$.

It is interesting to establish the first sound velocity $c$ from the obtained $\nu_{0}$ in superfluid ${ }^{4} \mathrm{He}$ making use of Bogoliubov equation:

$$
c=\sqrt{\frac{\rho \nu_{0}}{m}} .
$$

We shall obtain the value of $c$ at $291 \mathrm{~m} / \mathrm{s}$ whereas its experimental value $c^{e x p}$ equals $237 \mathrm{~m} / \mathrm{s}$. The first correction to (9) (see [6]) leads to $c=231 \mathrm{~m} / \mathrm{s}$ which is a very satisfactory result.

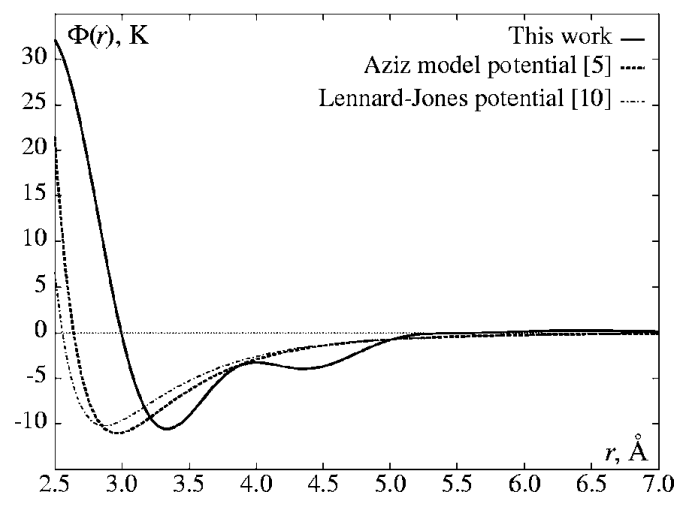

Fig. 3. The potential of interatomic interaction, comparison with model potentials.

Fig. 3 shows Aziz model potential for the sake of comparison [5] together with Lennard-Jones potential [10]. The potential in two approximations is presented in Fig. 4. Due to the restriction of experiment only the finite maximum value of the wave vector $q$ is available for carrying out the calculation in (7). Therefore, the numerical results for $\Phi(r)$ at small distances should not be considered as quite correct just as the "waves" at $r \simeq 2 \AA$. 


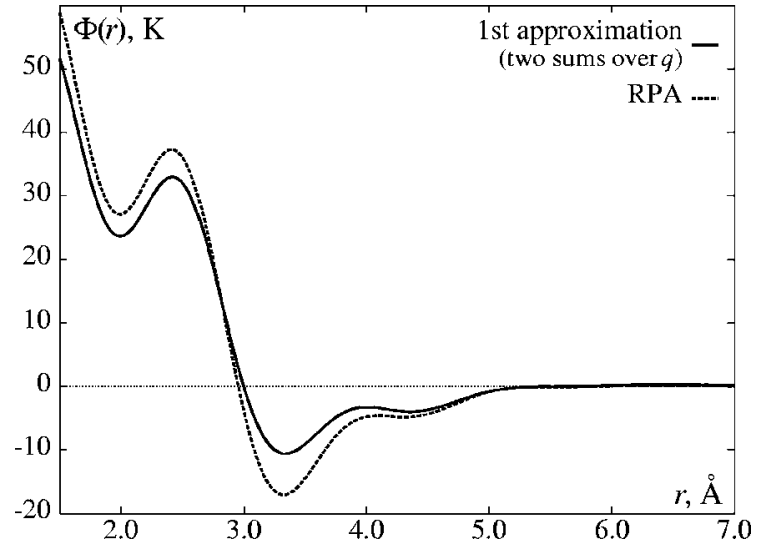

Fig. 4. The potential of interatomic interaction, comparison of approximations.
Partly they are the consequence of the experimental restriction mentioned above and partly they should be eliminated by taking into account many-particle effects. On the other hand, one can consider those $\Phi(r)$ values as non-significant since their contribution into the statistical operator is negligible in the temperature region where ${ }^{4}$ He stays liquid.

As can be seen from Fig. 4 and Table 3 the approximation of the two sums over the wave vector tangibly improves the value for the well depth of the potential leaving almost intact the position of the potential minimum (the distances between the atoms being equally balanced) in comparison with the random phase approximation. Otherwise stated, the influence of three- and four-particle correlations proves to be considerable.

The findings obtained in this paper are to be used for developing a theory from the first principles.

\section{APPENDIX}

\begin{tabular}{|c|c||c|c||c|c||c|c|}
\hline \hline$q, \AA^{-1}$ & $S_{q}$ & $q, \AA^{-1}$ & $S_{q}$ & $q, \AA^{-1}$ & $S_{q}$ & $q, \AA^{-1}$ & $S_{q}$ \\
\hline \hline 0.00 & 0.000 & 1.65 & 0.775 & 3.30 & 0.949 & 4.95 & 0.989 \\
0.05 & 0.017 & 1.70 & 0.865 & 3.35 & 0.954 & 5.00 & 0.988 \\
0.10 & 0.033 & 1.75 & 0.965 & 3.40 & 0.959 & 5.05 & 0.987 \\
0.15 & 0.053 & 1.80 & 1.069 & 3.45 & 0.964 & 5.10 & 0.986 \\
0.20 & 0.069 & 1.85 & 1.172 & 3.50 & 0.969 & 5.15 & 0.985 \\
0.25 & 0.084 & 1.90 & 1.263 & 3.55 & 0.973 & 5.20 & 0.985 \\
0.30 & 0.098 & 1.95 & 1.334 & 3.60 & 0.978 & 5.25 & 0.985 \\
0.35 & 0.111 & 2.00 & 1.377 & 3.65 & 0.983 & 5.30 & 0.985 \\
0.40 & 0.123 & 2.05 & 1.387 & 3.70 & 0.988 & 5.35 & 0.986 \\
0.45 & 0.134 & 2.10 & 1.365 & 3.75 & 0.992 & 5.40 & 0.986 \\
0.50 & 0.145 & 2.15 & 1.330 & 3.80 & 0.997 & 5.45 & 0.987 \\
0.55 & 0.155 & 2.20 & 1.290 & 3.85 & 1.001 & 5.50 & 0.988 \\
0.60 & 0.165 & 2.25 & 1.248 & 3.90 & 1.005 & 5.55 & 0.989 \\
0.65 & 0.175 & 2.30 & 1.203 & 3.95 & 1.009 & 5.60 & 0.990 \\
0.70 & 0.184 & 2.35 & 1.157 & 4.00 & 1.012 & 5.65 & 0.991 \\
0.75 & 0.194 & 2.40 & 1.116 & 4.05 & 1.014 & 5.70 & 0.992 \\
0.80 & 0.204 & 2.45 & 1.085 & 4.10 & 1.016 & 5.75 & 0.993 \\
0.85 & 0.215 & 2.50 & 1.061 & 4.15 & 1.018 & 5.80 & 0.994 \\
0.90 & 0.227 & 2.55 & 1.040 & 4.20 & 1.018 & 5.85 & 0.995 \\
0.95 & 0.241 & 2.60 & 1.021 & 4.25 & 1.018 & 5.90 & 0.995 \\
1.00 & 0.256 & 2.65 & 1.004 & 4.30 & 1.017 & 5.95 & 0.996 \\
1.05 & 0.273 & 2.70 & 0.989 & 4.35 & 1.016 & 6.00 & 0.997 \\
1.10 & 0.293 & 2.75 & 0.976 & 4.40 & 1.014 & 6.05 & 0.997 \\
1.15 & 0.315 & 2.80 & 0.965 & 4.45 & 1.012 & 6.10 & 0.998 \\
1.20 & 0.340 & 2.85 & 0.956 & 4.50 & 1.010 & 6.15 & 0.998 \\
1.25 & 0.368 & 2.90 & 0.949 & 4.55 & 1.007 & 6.20 & 0.999 \\
1.30 & 0.399 & 2.95 & 0.944 & 4.60 & 1.005 & 6.25 & 0.999 \\
1.35 & 0.433 & 3.00 & 0.941 & 4.65 & 1.002 & 6.30 & 0.999 \\
1.40 & 0.472 & 3.05 & 0.939 & 4.70 & 1.000 & 6.35 & 1.000 \\
1.45 & 0.515 & 3.10 & 0.939 & 4.75 & 0.997 & 6.40 & 1.000 \\
1.50 & 0.566 & 3.15 & 0.940 & 4.80 & 0.995 & 6.45 & 1.000 \\
1.55 & 0.626 & 3.20 & 0.942 & 4.85 & 0.993 & 6.50 & 1.000 \\
1.60 & 0.695 & 3.25 & 0.945 & 4.90 & 0.991 & 6.55 & 1.000 \\
\hline \hline
\end{tabular}

Table 2. Liquid helium-4 structure factor at $T=0 \mathrm{~K}$. 
I. O. VAKARCHUK, V. V. BABIN, A. A. ROVENCHAK

\begin{tabular}{|c|c|c|c|c|c|c|c|}
\hline$q, \AA^{-1}$ & $\nu_{q}, \mathrm{~K} \cdot \AA^{3}$ & $q, \AA^{-1}$ & $\nu_{q}, \mathrm{~K} \cdot \AA^{3}$ & $q, \AA^{-1}$ & $\nu_{q}, \mathrm{~K} \cdot \AA^{3}$ & $q, \AA^{-1}$ & $\nu_{q}, \mathrm{~K} \cdot \AA^{3}$ \\
\hline 0.00 & 1854.642 & 3.30 & 127.463 & 6.60 & 1.384 & 9.90 & 0.267 \\
\hline 0.10 & 1718.064 & 3.40 & 107.952 & 6.70 & 0.142 & 10.00 & 0.322 \\
\hline 0.20 & 1668.719 & 3.50 & 87.575 & 6.80 & -0.649 & 10.10 & 0.365 \\
\hline 0.30 & 1658.420 & 3.60 & 66.019 & 6.90 & -1.121 & 10.20 & 0.392 \\
\hline 0.40 & 1696.887 & 3.70 & 41.253 & 7.00 & -1.360 & 10.30 & 0.402 \\
\hline 0.50 & 1741.339 & 3.80 & 14.865 & 7.10 & -1.436 & 10.40 & 0.398 \\
\hline 0.60 & 1797.841 & 3.90 & -11.037 & 7.20 & -1.360 & 10.50 & 0.381 \\
\hline 0.70 & 1855.320 & 4.00 & -33.892 & 7.30 & -1.178 & 10.60 & 0.355 \\
\hline 0.80 & 1878.750 & 4.10 & -51.770 & 7.40 & -0.920 & 10.70 & 0.320 \\
\hline 0.90 & 1841.081 & 4.20 & -61.046 & 7.50 & -0.612 & 10.80 & 0.281 \\
\hline 1.00 & 1711.954 & 4.30 & -60.682 & 7.60 & -0.282 & 10.90 & 0.240 \\
\hline 1.10 & 1510.052 & 4.40 & -52.211 & 7.70 & 0.041 & 11.00 & 0.200 \\
\hline 1.20 & 1254.183 & 4.50 & -37.724 & 7.80 & 0.333 & 11.10 & 0.161 \\
\hline 1.30 & 985.829 & 4.60 & -18.491 & 7.90 & 0.575 & 11.20 & 0.127 \\
\hline 1.40 & 732.031 & 4.70 & 239 & 8.00 & 0.754 & 11.30 & 0.097 \\
\hline 1.50 & 492.149 & 4.80 & 30.105 & 8.10 & 0.869 & 11.40 & 0.072 \\
\hline 1.60 & 261.975 & 4.90 & 53.630 & 8.20 & 0.908 & 11.50 & 0.051 \\
\hline 1.7 & 95 & 5.00 & 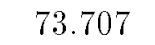 & 8.3 & 0.884 & 11.60 & 0.035 \\
\hline 1.80 & -62.007 & 5.10 & 89.292 & 8.40 & 0.807 & 11.70 & 0.023 \\
\hline 1.90 & -148 & 5.20 & 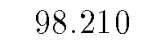 & . & 692 & 11.80 & 0.014 \\
\hline 2.00 & -193.077 & 5.30 & 100.844 & 8.60 & 0.554 & 11.90 & 0.008 \\
\hline 2.10 & -198 & 5.40 & 3 & . & 0.406 & 12.00 & 0.004 \\
\hline 2.20 & -178.040 & 5.50 & 90.167 & 8.80 & 0.263 & 12.10 & 0.001 \\
\hline 2.30 & -141. & 5.60 & 79.563 & 8.90 & 0.139 & 12.20 & -0.000 \\
\hline 2.40 & -85.980 & 5.70 & 67.075 & 9.00 & 0.040 & 12.30 & -0.001 \\
\hline 2.50 & -42.129 & 5.80 & 54.289 & 9.10 & -0.032 & 12.40 & -0.002 \\
\hline 2.60 & -1.734 & 5.90 & 42.231 & 9.20 & -0.067 & 12.50 & -0.002 \\
\hline 2.7 & 39.482 & 6.00 & 31.578 & 9.30 & -0.070 & 12.60 & -0.001 \\
\hline 2.80 & 78.124 & 6.10 & 22.495 & 9.40 & -0.046 & 12.70 & -0.001 \\
\hline 2.90 & 110.073 & 6.20 & 15.420 & 9.50 & 0.000 & 12.80 & -0.001 \\
\hline 3.00 & 130.602 & 6.30 & 10.042 & 9.60 & 0.061 & 12.90 & -0.001 \\
\hline 3.10 & 141.836 & 6.40 & 6.110 & 9.70 & 0.131 & 13.00 & -0.000 \\
\hline 3.20 & 140.580 & 6.50 & 3.337 & 9.80 & 0.202 & 13.10 & -0.000 \\
\hline
\end{tabular}

Table 3. The Fourier image of the interatomic potential in ${ }^{4} \mathrm{He}$. 


\begin{tabular}{|c|c|c|c|c|c|c|c|}
\hline$r, \AA$ & $\Phi(r), \mathrm{K}$ & $r, \AA$ & $\Phi(r), \mathrm{K}$ & $r, \AA$ & $\Phi(r), \mathrm{K}$ & $r, \AA$ & $\Phi(r), \mathrm{K}$ \\
\hline 0.00 & $\begin{array}{l}(229.852) \\
\end{array}$ & 3.80 & -4.250 & 7.70 & 0.041 & 11.50 & -0.039 \\
\hline 0.10 & $(222.056)$ & 3.90 & -3.487 & 7.80 & 0.050 & 11.60 & -0.038 \\
\hline 0.20 & 200.262 & 4.00 & -3.261 & 7.90 & 0.067 & 11.70 & -0.036 \\
\hline 0.30 & 168.745 & 4.10 & -3.406 & 8.00 & 0.092 & 11.80 & -0.033 \\
\hline 0.40 & 133.153 & 4.20 & -3.695 & 8.10 & 0.120 & 11.90 & -0.030 \\
\hline 0.50 & 99.024 & 4.30 & -3.917 & 8.20 & 0.150 & 12.00 & -0.028 \\
\hline 0.60 & 70.679 & 4.40 & -3.931 & 8.30 & 0.178 & 12.10 & -0.025 \\
\hline 0.70 & 50.694 & 4.50 & -3.685 & 8.40 & 0.199 & 12.20 & -0.022 \\
\hline 0.80 & 39.840 & 4.60 & -3.206 & 8.50 & 0.213 & 12.30 & -0.018 \\
\hline 0.90 & 37.253 & 4.70 & -2.579 & 8.60 & 0.216 & 12.40 & -0.014 \\
\hline 1.00 & 40.735 & 4.80 & -1.908 & 8.70 & 0.209 & 12.50 & -0.010 \\
\hline 1.10 & 47.228 & 4.90 & -1.287 & 8.80 & 0.192 & 12.60 & -0.005 \\
\hline 1.20 & 53.506 & 5.00 & -0.783 & 8.90 & 0.167 & 12.70 & -0.000 \\
\hline 1.30 & 56.975 & 5.10 & -0.422 & 9.00 & 0.137 & 12.80 & 0.005 \\
\hline 1.40 & 56.302 & 5.20 & -0.198 & 9.10 & 0.104 & 12.90 & 0.010 \\
\hline 1.50 & 51.624 & 5.30 & -0.082 & 9.20 & 0.071 & 13.00 & 0.014 \\
\hline 1.60 & 44.279 & 5.40 & -0.034 & 9.30 & 0.041 & 13.10 & 0.018 \\
\hline 1.70 & 36.209 & 5.50 & -0.017 & 9.40 & 0.016 & 13.20 & 0.021 \\
\hline 1.80 & 29.316 & 5.60 & -0.004 & 9.50 & -0.003 & 13.30 & 0.023 \\
\hline 1.90 & 24.954 & 5.70 & 0.020 & 9.60 & -0.014 & 13.40 & 0.025 \\
\hline 2.00 & 23.656 & 5.80 & 0.058 & 9.70 & -0.020 & 13.50 & 0.025 \\
\hline 2.10 & 25.074 & 5.90 & 0.107 & 9.80 & -0.019 & 13.60 & 0.025 \\
\hline 2.20 & 28.115 & 6.00 & 0.159 & 9.90 & -0.015 & 13.70 & 0.024 \\
\hline 2.30 & 31.250 & 6.10 & 0.204 & 10.00 & -0.009 & 13.80 & 0.023 \\
\hline 2.40 & 32.953 & 6.20 & 0.237 & 10.10 & -0.003 & 13.90 & 0.022 \\
\hline 2.50 & 32.134 & 6.30 & 0.256 & 10.20 & 0.003 & 14.00 & 0.020 \\
\hline 2.60 & 28.437 & 6.40 & 0.262 & 10.30 & 0.007 & 14.10 & 0.018 \\
\hline 2.70 & 22.283 & 6.50 & 0.256 & 10.40 & 0.008 & 14.20 & 0.016 \\
\hline 2.80 & 14.660 & 6.60 & 0.243 & 10.50 & 0.006 & 14.30 & 0.013 \\
\hline 2.90 & 6.784 & 6.70 & 0.224 & 10.60 & 0.002 & 14.40 & 0.010 \\
\hline 3.00 & -0.238 & 6.80 & 0.203 & 10.70 & -0.005 & 14.50 & 0.007 \\
\hline 3.10 & -5.631 & 6.90 & 0.180 & 10.80 & -0.012 & 14.60 & 0.003 \\
\hline 3.20 & -9.036 & 7.00 & 0.156 & 10.90 & -0.019 & 14.70 & 0.000 \\
\hline 3.30 & -10.486 & 7.10 & 0.131 & 11.00 & -0.026 & 14.80 & -0.003 \\
\hline 3.40 & -10.320 & 7.20 & 0.107 & 11.10 & -0.032 & 14.90 & -0.005 \\
\hline 3.50 & -9.064 & 7.30 & 0.084 & 11.20 & -0.036 & 15.00 & -0.007 \\
\hline 3.60 & -7.308 & 7.40 & 0.064 & 11.30 & -0.039 & 15.10 & -0.007 \\
\hline 3.70 & -5.580 & 7.50 & 0.049 & 11.40 & -0.040 & 15.20 & -0.007 \\
\hline
\end{tabular}

Table 4 . The potential of the interatomic interaction in ${ }^{4} \mathrm{He}$. 
[1] Physics of Simple Liquids, edited by H. N.V. Temperley, J. S. Rowlison, G. S. Rushbrooke, (Wiley Interscience Division, New York, 1968) [Translated into Russian (Mir, Moscow, 1971)].

[2] C. A. Croxton, Liquid State Physics - A Statistical Mechanical Introduction (Cambridge University Press, 1974) [Translated into Russian (Mir, Moscow, 1978)].

[3] R. A. Aziz, V. P. S. Nain, J. S. Carley, W. L. Taylor, G. T. McConville, J. Chem. Phys. 70, 4430 (1979).

[4] R. A. Aziz, M. J. Slaman, A. Koide, A. R. Allnatt, W. J. Meath, Mol. Phys. 77, 321 (1992).
[5] A. R. Janzen, R. A. Aziz, J. Chem. Phys. 107, 914 (1997).

[6] I. A. Vakarchuk, I. R. Yukhnovskii, Teor. Mat. Fiz. 40, 100 (1979); 42, 112 (1980).

[7] I. A. Vakarchuk, Teor. Mat. Fiz. 80, 439 (1989); 82, 438 (1990).

[8] E. C. Svensson, V. F. Sears, A. D. B. Woods, P. Martel, Phys. Rev. B 21, 8 (1980).

[9] N. N. Bogoliubov, Lektsii z kvantovoi statystyky (Lectures on Quantum Statistics) (Kyiv, 1949).

[10] W. L. McMillan, Phys. Rev. A 138, 442 (1965).

\title{
ПОТЕНЦІЯЛЬНА ЕНЕРІІЯ ВЗАЕМОДІї МІЖ АТОМАМИ ГЕЛІЮ
}

\author{
I. О. Вакарчук, В. В. Бабін, А. А. Ровенчак \\ Лъвівсъкий національний університет імені Івана Франка \\ Кафедра теоретичноӥ фізики \\ вул. Драгоманова, 12, 79005, Львів, Украӥна
}

$\mathrm{V}$ роботі з перших принципів та без використання допоміжних параметрів отримано парний потенпіял міжатомної взаємодії для гелію. При цьому застосовано знайдені раніше з рівняння ІШредингера співвідношення, які пов'язують Фур'є-зображення потенпіялу з коефіпієнтними функџіями в розклапі логарифма хвильової функції основного стану багатобозонної системи за степенями флюктуацій густини. Розглянуті RPA-наближення як нульова та наближення “двох сум за хвильовим вектором” як перша апроксимащія.

3 результатів роботи випливає значення швидкости першого звуку $231 \mathrm{~m} / \mathrm{c}$, тоді як експеримент дає 237 м/с. Узгодження нашого потенпіялу з модельним потенпіялом Азіза $є$ пілком задовільним: глибини потенпіяльних ям становлять відповідно $-10.58 \mathrm{~K}$ та $-11.04 \mathrm{~K}$, точки мінімуму - $3.34 \AA$ та $2.99 \AA$. 